تأثير النسميد بـ NPK في مياه الري Fertigation علي نمو وصفات أصناف من الورد النامي تحت

$$
\text { ظُروف البيوت رفدان المجهوج القحطاني } 1
$$

(Armitage and Laushman, 2003) الأسمدة المتوازنة لزيادة الإنتاج و تحسين النمو في الورود. تغذية نباتية الغير كافية يسبب اضطرابات خطيرة قد تؤدي في النهاية إلي الخفاض في نمو النبات وكمية المحصول. يشجع إضافة النتروجين (N) والفوسفور (P) والبوتاسيوم (K) النمو الخضري وأيضا يساعد النبات إثناء فترة التفتح الزهري وتشجيع العمليات المرتبطة ببدء التزهير

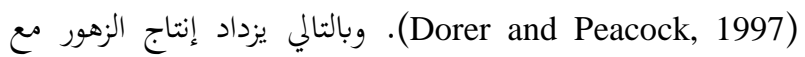
N. P. K. زيادة المستوي الأمثل من .(Young et al., 1976, Umma and Gowda, 1986) تؤدي الكميات الكثيرة منNPK التي تضاف علي السطح خسائر في المحاصيل بسبب عدم كفاءة استخدام النبات للأسمدة

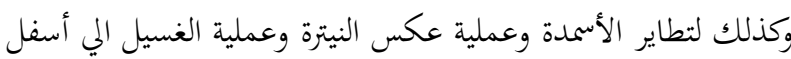

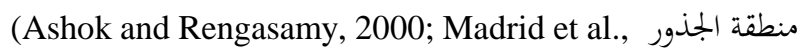
2001; Mohammad and Zuraiqi, 2003). المغذيات يسبب أيضا تلوث بيئي الذي يؤثر علي جميع أنواع الحياة

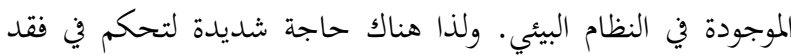

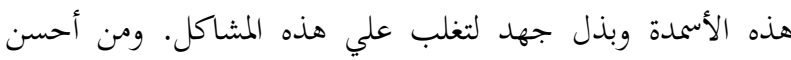

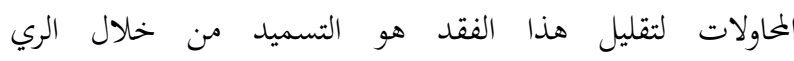
(Fertigation)

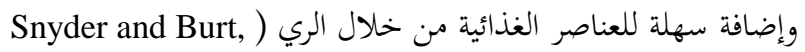
1976). التسميد من خلال الري يجمع بين أثنين من الاحتياجات الأساسية لنمو النبات وتطويره مثل الماء والمغذيات. الخلط الصحيح بين الماء والمغذيات هي مفتاح الكمية والجودة العالية من المصصول. هذه التقنية (إضافة السماد مع الري) ذات مرونة وفعالية من حيث

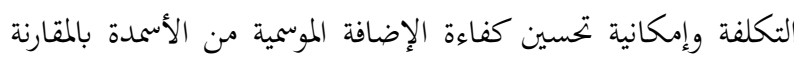
بطرق الإضافة التقليدية للأسمدة (Jaynes et al., 1992). في دراسة

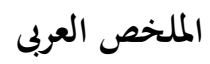

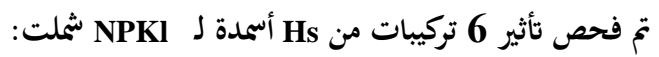

$(100 \mathrm{~N}+20 \mathrm{P}+100 \mathrm{~K}(\mathrm{~T} 1), 125 \mathrm{~N}+30 \mathrm{P}+130 \mathrm{~K}(\mathrm{~T} 2), 150 \mathrm{~N}+40 \mathrm{P}+16$ 0K (T3), 175N+50P+190K (T4), 200N+60P+220K (T5) ،225N+70P+250K (T6) ppm)

المضاف في مياه الري (Fertigation) وكان الري علي فترات كل

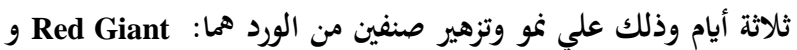

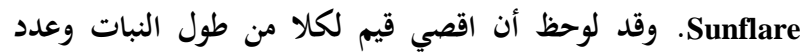

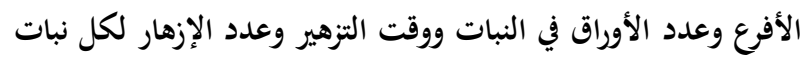

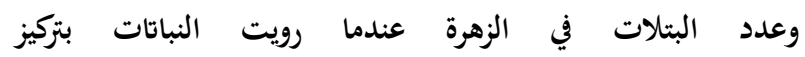
175N+50P+190K

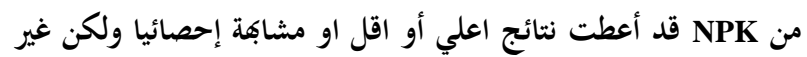

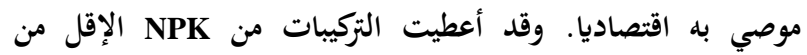
مستوي التركيب 175N+50P+190K جزء في المليون في مياه الري صفات نباتية إقل إحصائيا وبالتالي غير موصي بها اقتصاديا كمركبات مثالية.

\section{المقدمة والمشكلة البحثية}

اكتسبت الورد إهمية اقتصادية كبيرة بسبب قيمتها التسويقية العالية

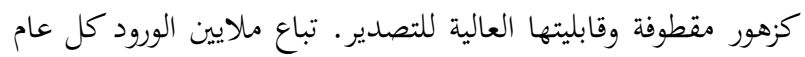
ويزداد الطلب علي هذه الورود المقطوفة بشكل كبير عبر الأثير (Yousuf and Dennis, 1999) بالكامل. وهناك حاجة لإقامة هذه المشاريع علي أسس حديثة وإجراء بكوث واسعة النطاق في جميع جوانب تقنية إنتاج الورد والتي قد تشمل اختيار وتربية أصناف ذات قيمة عالية ومكافحة الآفات والإمراض وعمليات التقليم وإدارة المحاصيل بما في ذلك التغذية و تقنية

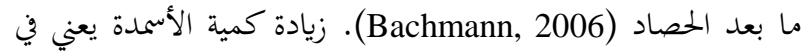
الواقع النجاح وزيادة المحصول من خلال زيادة عدد وحجم الزهور

1

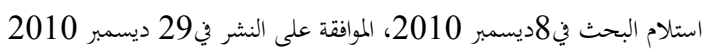


الهفوف- قطر السريع. زرعت نباتات الورود في اصص بها رمل مخلوط مع بتموس بنسبة 1:1 حيث رويت بمياه الري المحتوية علي تركيبات مختلفة من النتروجين (100، 125، 150، 150، 175، بندبة 200 و 225

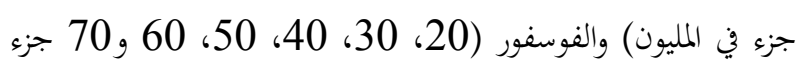
في المليون) والبوتاسيوم (100، 130، 130، 160، 190، 190، 220 و250جزء في المليون) علي فترات كل ثلاثة أيام. والتالي الستة معاملات المستخدمة في التجربة معبرا عن التركيز في مياه الري بالجزء فئري في المليون:

T1 $\quad(\mathrm{NPK}=100+20+100)$

$\mathrm{T} 2 \quad(\mathrm{NPK}=125+30+130)$

T3 $\quad(\mathrm{NPK}=150+40+160)$

T4 (NPK $=175+50+190)$

T5 $\quad(\mathrm{NPK}=200+60+220)$

T6 $\quad(\mathrm{NPK}=225+70+250)$

استخدمت اسمدة اليوريا (46\% نتروجين) والسوبر فوسفات

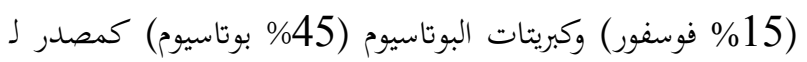
N, P, K السكون بحيث يكون طولها 15سم من سطح بيئة النمو. وفي إثناء الدراسة سجلت الصفات التالية في كل معاملة وهي طول النبات (سم) وعدد الأفرع في كل نبات وعدد الأوراق في كل نبات وعدد الأيام لظهور أول زهرة وعدد الأزهار في النبات الواحد وعدد وعد البتلات في كل زهرة. صممت التجربة بتصميم التجارب العاملية في عاملين

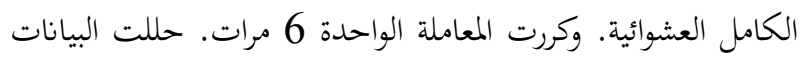

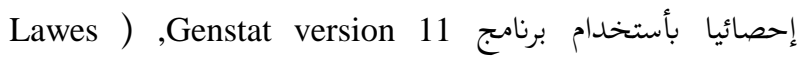
Agricultural Trust, Rothamsted Experimental Station,

(UK, 2008

\section{النتائج والمناقشات}

وضحت نتائج الصفات المدروسة عن وجود فروق معنوية عالية

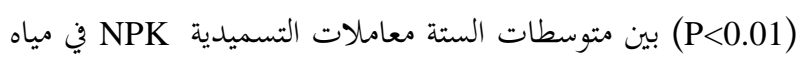
الري وبين صنفي الورد (Red Giant and Sunflare). أشار الشكل1 أن المعاملة T4 قد أعطت أقصي ارتفاع لنباتات صنفي الورد وقد قل الطول بنسبة 20\% في صنف Red Giant وبنسبة 19 فعاملة

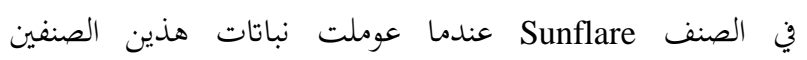
المعاملةT1. وقد قل أيضا طول النبات بنسبة 16\% في صنف Red
علي أصناف الورد قد أعطيت الإضافة التركيبية من 175جزء في المليون من N و M و50 وجزء في المليون من P اعلي عدد من الإزهار و

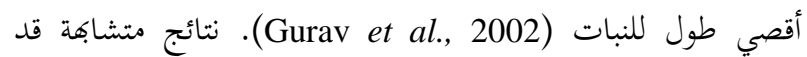
تحصل عليها في تحارب علي صنف First Red عندما سمدت النباتات

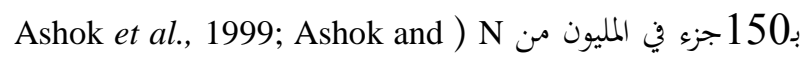

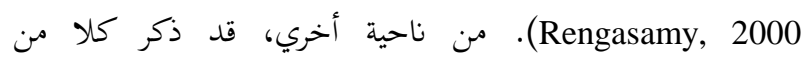
Armitage and Jawjita في المليون من N قد أنتجت اعلي مصول من زهور ولكن بسبب تحسن النمو والاستجابة للتطور للورد فأن تركيز 300 جزء في في المليون

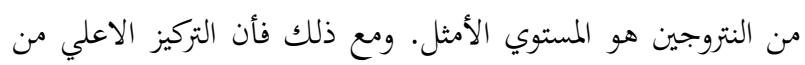
النتروجين (500 جزء في المليون) قد قلل معنويا نمو ونوعية الأزهار. كما ذكر نتائج مماثلة علي العديد من نباتات الزينة مثل زهرة الاقحوان

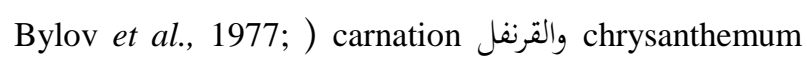
Komosa, 1979; Krishna et al., 1999; Krishna and .(Gowda, 2000; Goto et al., 2001

أجريت قليل من تجارب التسميد بالري في المملكة العربية

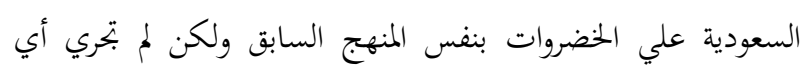
دراسة عن تأثير التسميد بالري علي الورود أو نباتات الزينة الاخري.

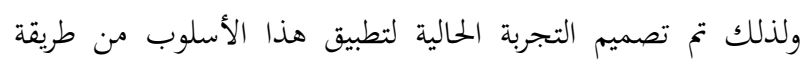

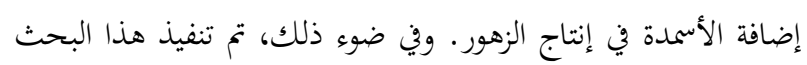

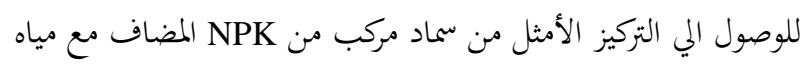
الري(Fertigation) للحصول علي نمو جيد وتحسين الإنتاج لنوعين من الورود (Red Giant and Sunflare) تحت الظروف المناخية الزراعية في المملكة العربية السعودية.

\section{المواد وطرق البحث}

الهدف الاساسي من هذا البحث التجربة هو تقييم تأثير

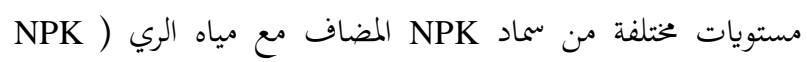
(fertigation hybrids tea rose cultivars viz. 'Red Giant' and 'Sunflare' أجريت هذه التجربة في احدي البيوت المحمية المجهزة والمتحكم فيه بيئيا

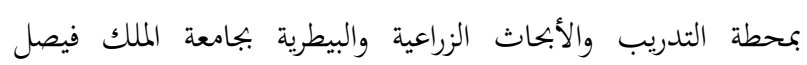
بالإحساء بالمملكة العربية السعودية. تقع مطة التدريب والأبحاث

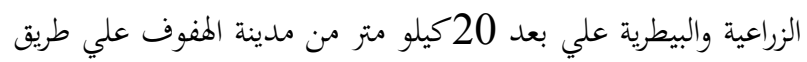


Ashok and Rengasamy (2000) سماد النتروجيني في مياه الري تنتج أكبر عدد من الأوراق للنبات في الورود. أظهرت نتائج هذا البحث أن النباتات قد أخذت وقتا أقل في تفتح الزهرة الأولي عندما عرضت للمعاملةT4 يليها في التأثير المعاملة

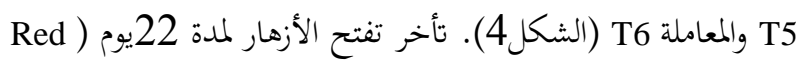
(Giant) و16 يوم (Sunflare) عندما عوملت هذه الأصناف للتركيز

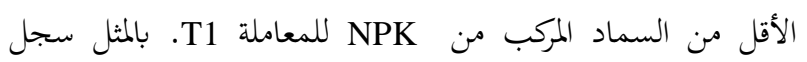
16 يوم في الصنف (Red Giant) و12 يوم في الصنف (Sunflare) لتفتح الزهرة الأولي عندما رويت النباتات بالمعاملة T2 يليها نباتات المعاملة T3 حيث كان 14 يوم (Red Giant) و10 أيام (Sunflare) تأخر في التزهير قد لوحظ بالمقارنة بالمعاملة T4. هذه النتائج متوافقة

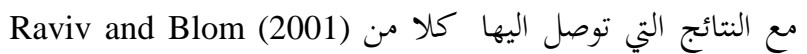
and Palai et al. (2002)

عندما أضيف سماد NPK مع مياه الري حقنا (Fertigation). يوضح الشكل 5 أن العدد الاقصي للإزهار في النبات الواحد (14 في الصنف Red Giant و 11 في الصنف دunflare) كان في النباتات التي تعرضت للمعاملة T4. نتائج متشاهة قد تحصل عليها

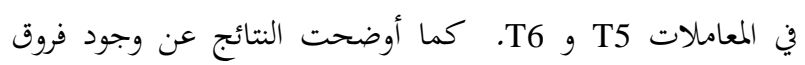

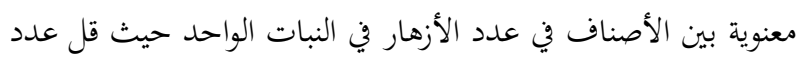

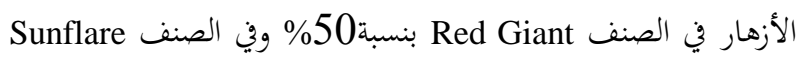
بنسبة 55\% تحت تأثير المعاملتين T1 وT2. ونتائج وقد تحصل عليها Gurav et al. (2002) لإزهار في النبات الواحد عندما رويت النباتات بمياه بها تركيز 175 جزء في المليون من كلا من النتروجين والبوتاسيوم وتركيز الفوسفور 0

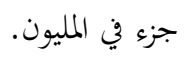

بالنسبة لعدد البتلات في الزهرة الواحدة في كلا الصنفين(شكل 6). النباتات التي رويت بالمعاملة T4 أعطت أكبر عدد من البتلات في كل زهرة حيث كان هذا العدد 24 في الصنف Red Giant و35 بن 35

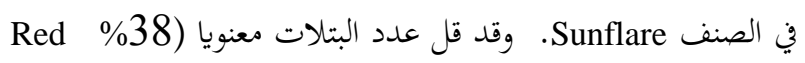

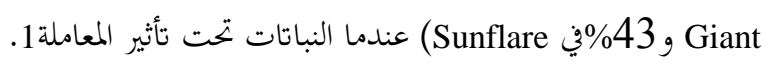

Giant و بنسبة 15\% في نباتات الصنف Sunflare عندما تعرضت النباتات في هذين الصنفين للمعاملة T2 ويليها نباتات عوملت T3

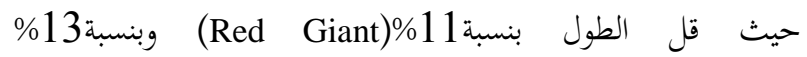
(Sunflare) تماثل إحصائيا مع نباتات المعاملة T4. نتائج مماثلة قد تحصل عليها Montezuma Palai et al. (2002) المعاملة بـ NPK في مياه الري بتركيز 200K +300P+300N جزء في المليون. وبالمثل، كان أقصي طول في نباتات القرنفل قد تحصل بـرديز

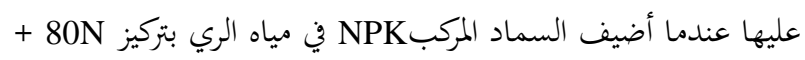
120K + 100P جزء في المليون (Krishna et al. 1999). بالمثل، كان أقصي عدد الأفرع بالنسبة للنبات الواحد (14 في صنف Red Gaint و 11 في الصنف Sunflare ) عندما كان تركيز 175N+50P+190K في مياه الري المضاف للنباتات بمعدل NPK جزء في المليون (T4). التركيز الاقل من NPK المضاف مع مياه الري

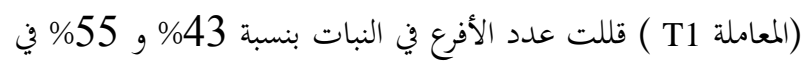
الصنف Red Giant و الصنف Sunflare علي التوالي. المعاملات الاخري مثل T2 خفضت عدد الأفرع في النبات الواحد لأكثر من

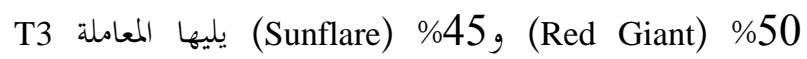

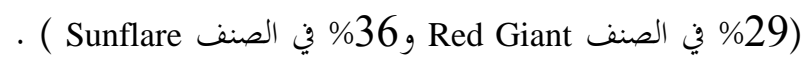

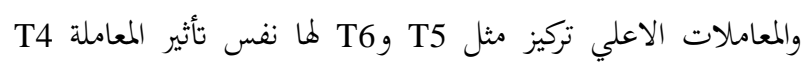
Veeranna et al. علي متوسط عدد الأفرع في النبات. وقد سجريز Capsicum (2000) عندما أضاف 150جزء في المليون من سماد البوتاسيوم في مياه الري. وأعطت المعاملة T4 اكبر عدد من الأوراق في النبات حيث كان

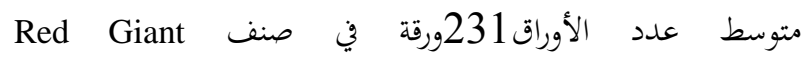
و207ورقة في الصنف Sunflare والذي كان متساويا إحصائيا مع تأثير المعاملة T5 والمعاملة T6 (شكل 3) ول. وقد نقص عدد الأوراق في النبات بنسبة 20\% في كلا الصنفين تحت الدراسة عندما عوملت النباتات بالمستوي الأقل في التركيز من سماد NPK (T1) يليه المعاملة

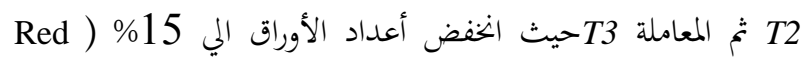
(Red Giant) \%14， (Sunflare)\%14， (Giant

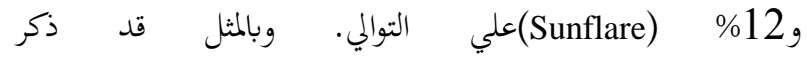




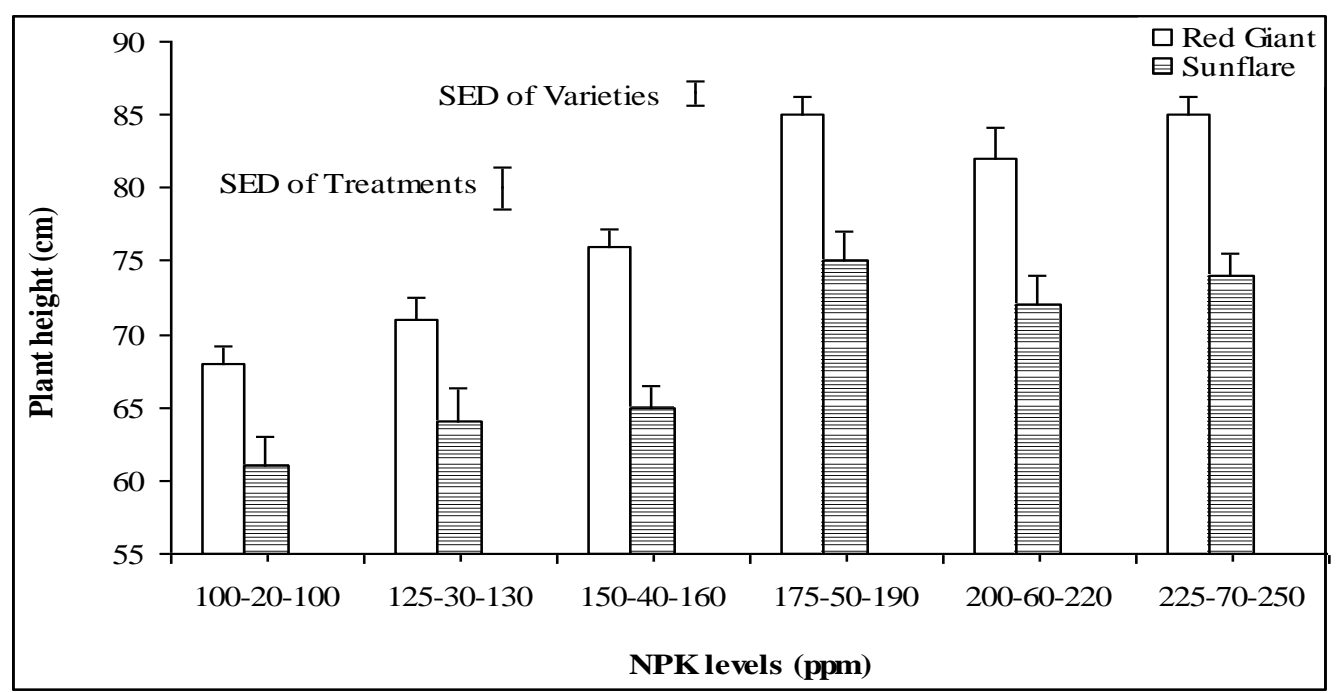

شكل. 1أثير تركيزات مختلفة من NPK المضافة مع مياه الري (NPK levels, ppm) علي طول نبات الورد (سم) ( Plant) (العمود الأبيض) والصنف Sed Giant (العمود المظلل).تعني Sunflare (العنف SED of treatment, cm القياسي للفرق بين متوسطات المعاملات وSDE of Varietiesتعني الخطأ القياسي بين الأصناف عند مستوي احتمالية 5\%. الأخطاء القياسية التي أكبر من العلامات الموضحة بأطوال علي المربعات تمثل الاختلافات داخل المكررات

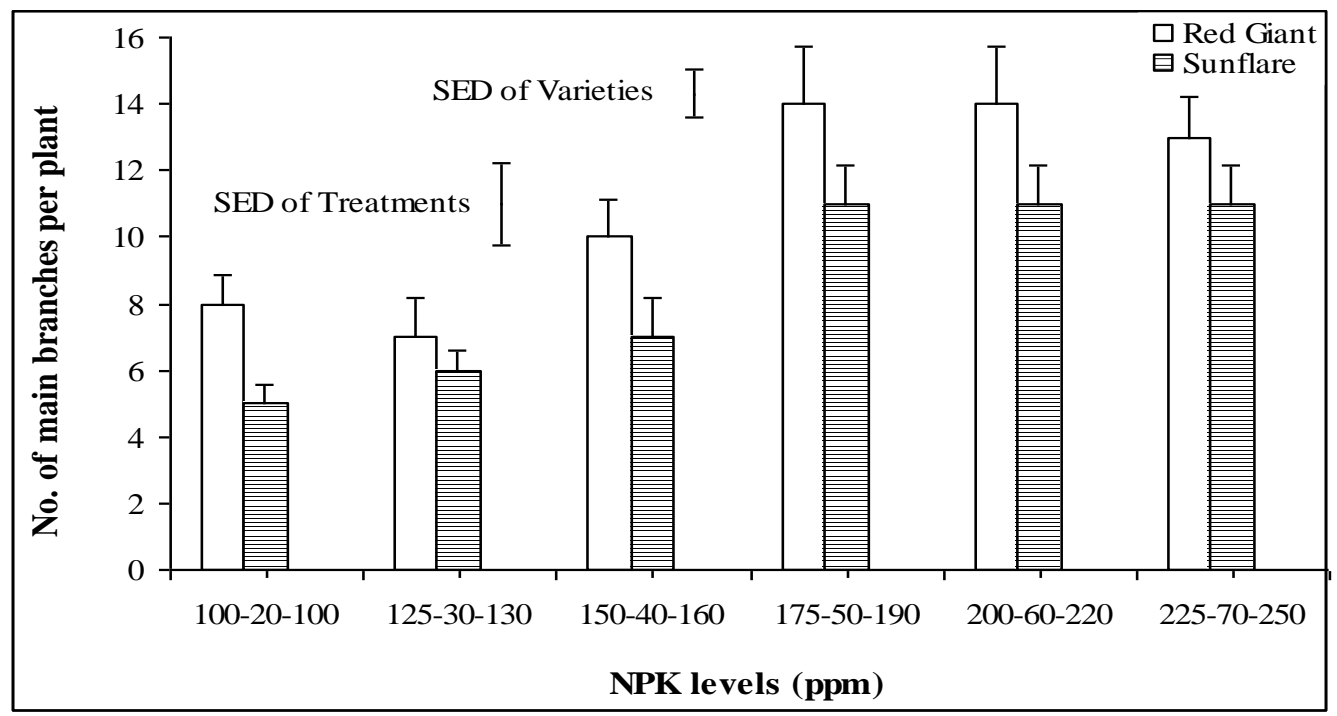

شكل 2. تأثير تركيزات مختلفة من NPK المضافة مع مياه الري (NPK levels, ppm) علي متوسط عدد الأفرع /نبات No. of (main branches per plant SED of treatment مستوي احتمالية 5\%. الأخطاء القياسية التي أكبر من العلامات الموضحة بأطوال علي المربعات تمثل الاختلافات داخل المكررات. 


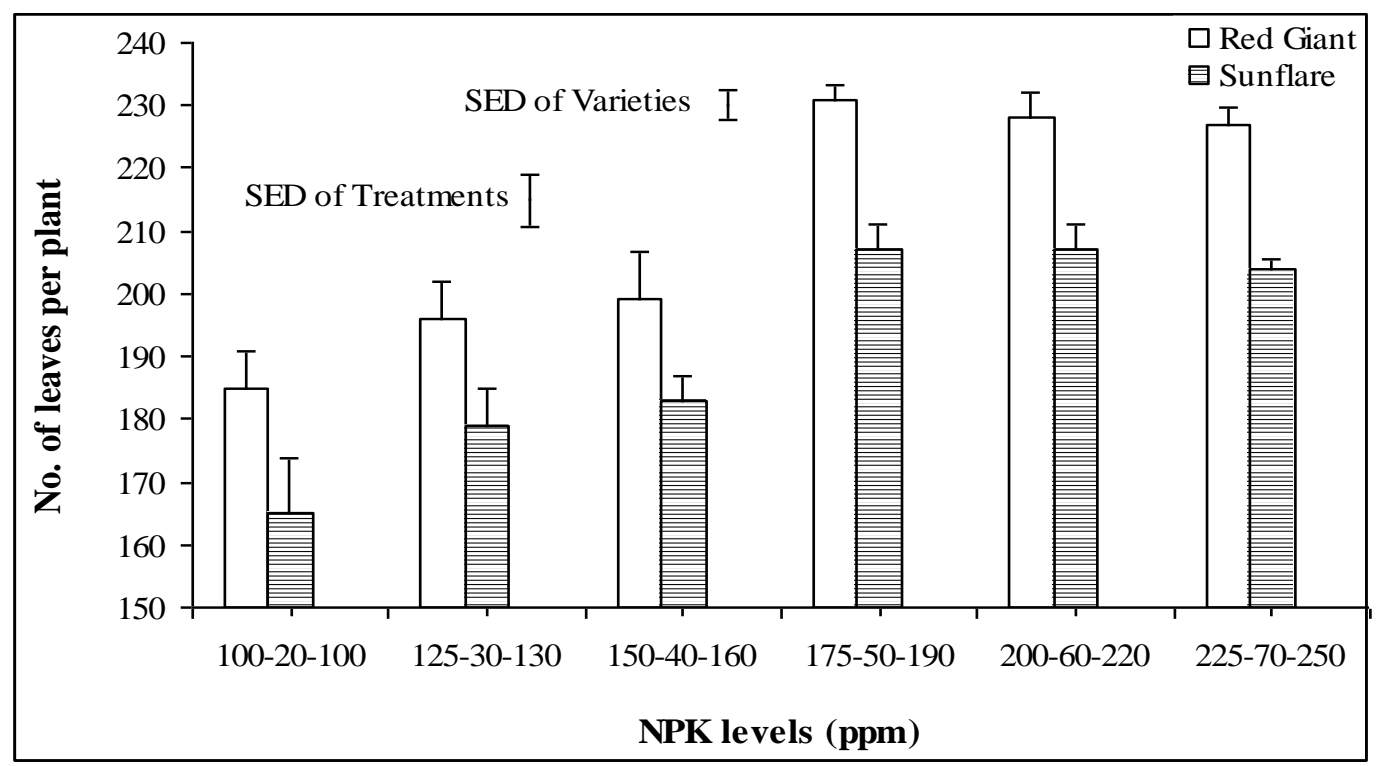

شكل3. تأثير تركيزات مختلفة من NPK المضافة مع مياه الري (NPK levels, ppm) علي عدد الأوراق / نبات ( No. of leaves)

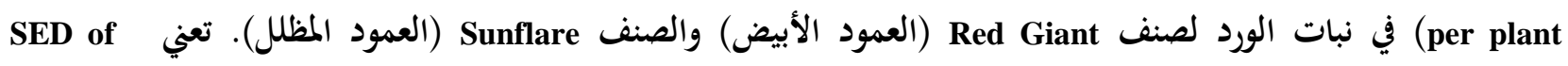
treatment احتمالية 5\%٪. الأخطاء القياسية التي أكبر من العلامات الموضحة بأطوال علي المربعات تمثل الاختلافات داخل المكررات.

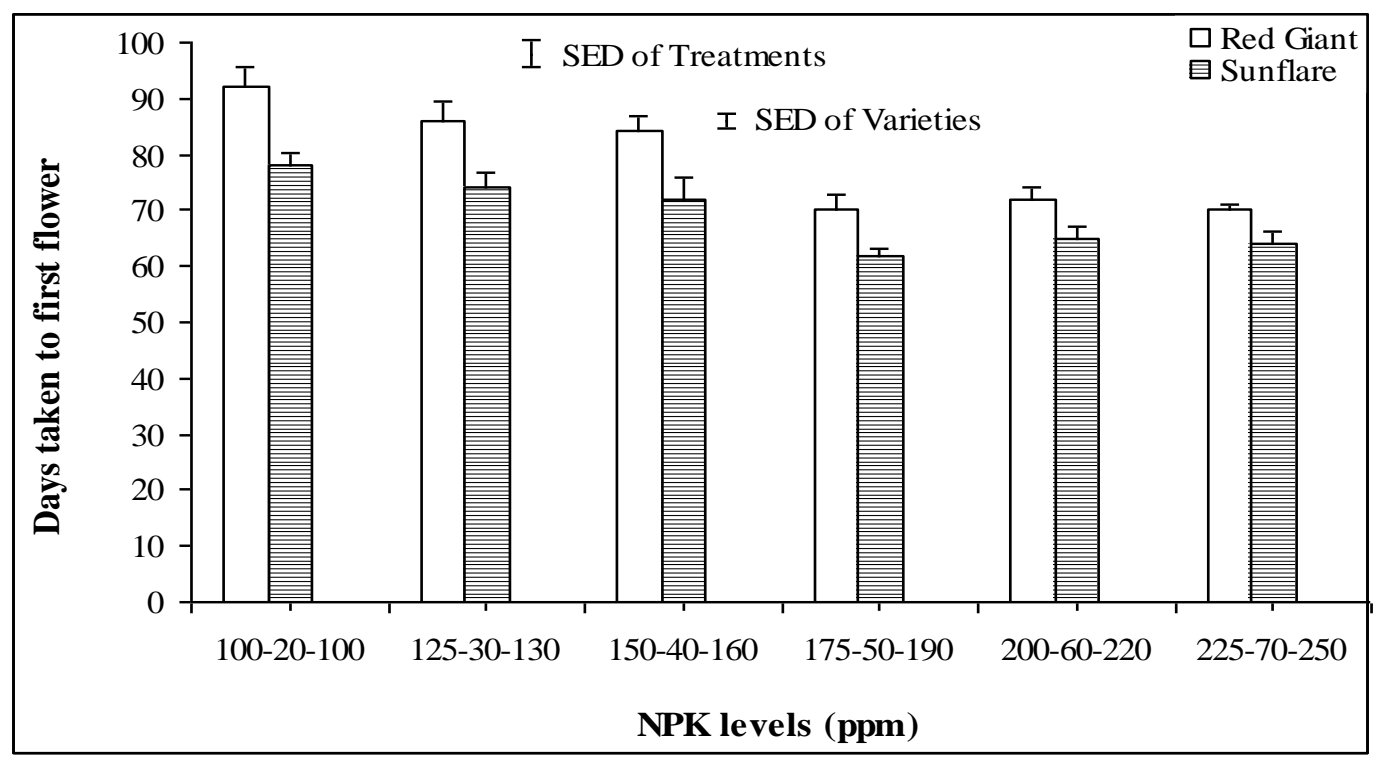

شكل4. تأثير تركيزات مختلفة من NPK المضافة مع مياه الري (NPK levels, ppm) علي عدد أيام ظهور أول زهرة ( Days)

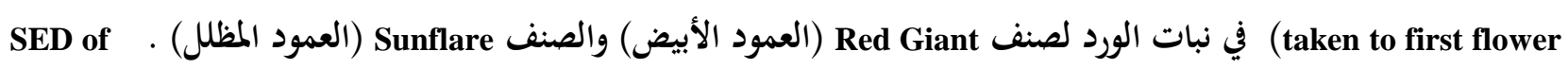
treatment مستوي احتمالية 5\% الأخطاء القياسية التي أكبر من العلامات الموضحة بأطوال علي المربعات تمثل الاختلافات داخل 


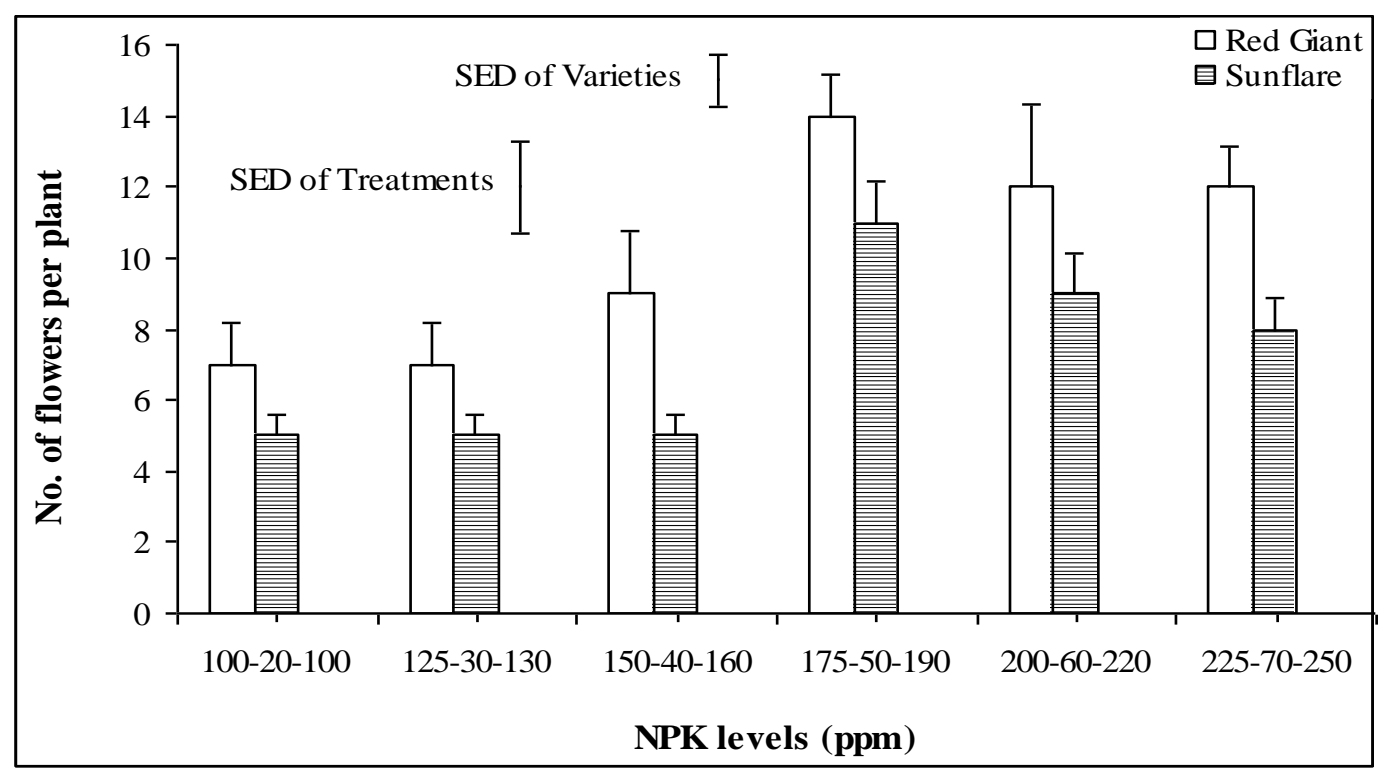

شكل5. ثأثير تركيزات مختلفة من NPK المضافة مع مياه الري (NPK levels, ppm) علي عدد الإزهار / نبات ( No. of flower) SED of في نer plant treatment الخطأ القياسي للفرق بين متوسطات المعاملات وتعني SDE of Varieties الخطأ القياسي بين الأصناف عند مستوي احتمالية 5\% الأخطاء القياسية التي أكبر من العلامات الموضحة بأطوال علي المربعات تمثل الاختلافات داخل المكررات.

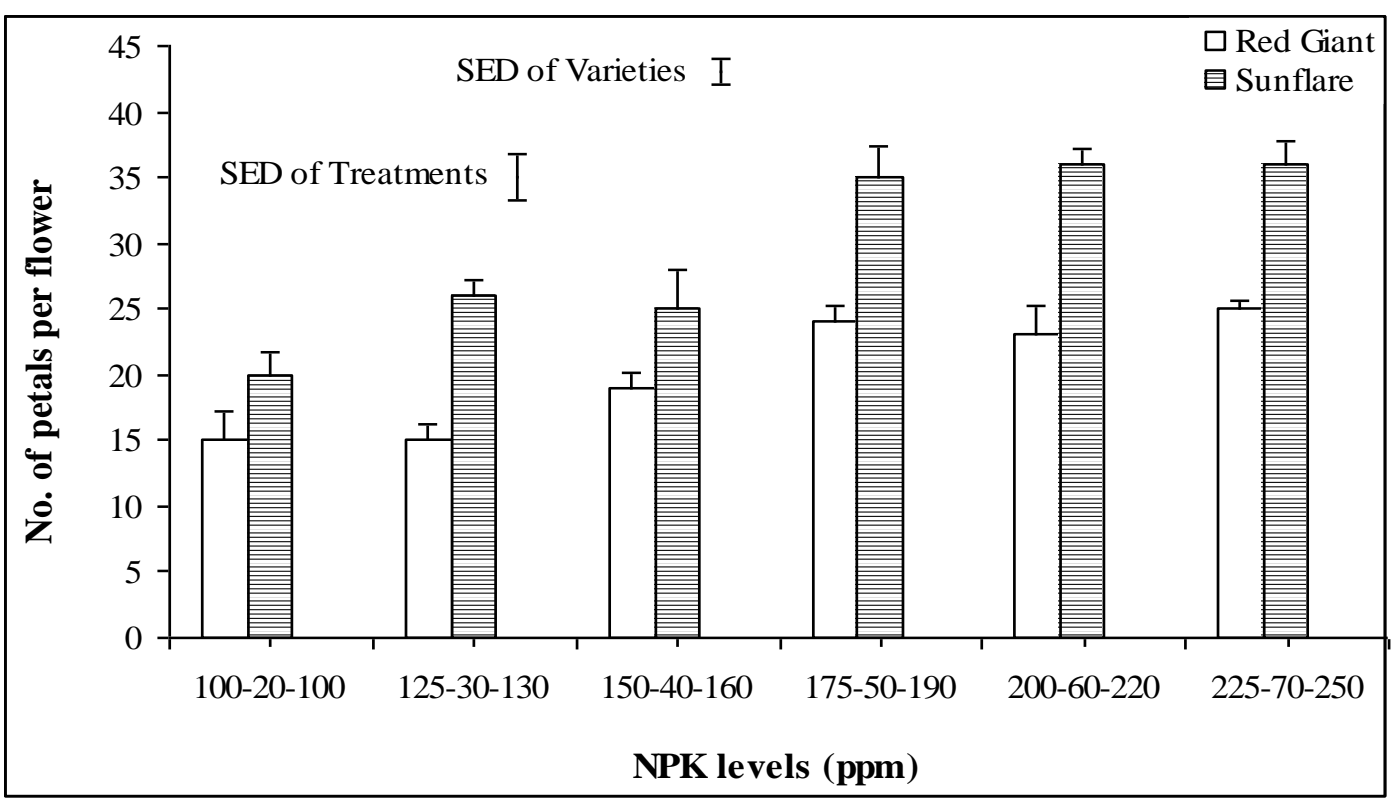

شكل 6. تأثير تركيزات مختلفة من NPK المضافة مع مياه الري (NPK levels, ppm) علي عدد البتلات/ زهرة في نبات الورد لصنف Red Giant (العمود الأبيض) والصنف Sunflare (العمود المظلل) .تعني SED of treatment الخطأ القياسي للفرق بين متوسطات المعاملات وتعني SDE of Varieties الحطأ القياسي بين الأصناف عند مستوي احتمالية 5\%. الأخطاء القياسية التي أكبر من العلامات الموضحة بأطوال علي المربعات تمثل الاختلافات داخل المكررات. 
Dorer, S. and C. Peacock. 1997. The effects of humate and organic fertilizer on establishment and nutrition of creeping bentgrass putting green. J. Intl. Turf. Res. Soc., 437-443.

Genstat version 11.,2008. Lawes Agricultural Trust, Rothamsted Experimental Station, UK, Goto, T., N. Takaya, N. Yoshioka, Y. Yoshida, Y. Kageyama and K. Konishi. 2001. Effects of water and nutrient stresses on reduction of vegetative growth in chrysanthemum grown under restricted root zone volume. J. Japanese Soc. Hort. Sci., 70(6): 760-766

Gurav, S.K., S. Patel. M. Patel. B. Singh R. Misraand S. Misra. 2002. Fertigation of roses under natural ventilated Poly house conditions. Flori. Res. Trend in India, 222223.

Jaynes, D., R. Rice and D. Hunsaker. 1992. Solute transport during chemigation of level basin. Transaction of the ASAE., 35(6): 1809-1815.

Komosa, A. 1979. Indices of nitrogen and potassium nutrition in chrysanthemum cv. 'Balcambe Perfection'. Nauk Ral. I Komis Nauk Les. PTPN, 47: 159-168. (Hort. Absts., 50(5): 292-193, 1980).

Krishna, B.K. and M. Gowda. 2000. Nutrient accumulation and uptake in carnation cultivars grown in poly house as affected by fertigation. Uni. Agric. Sci. Bangalore. 29(11): 181-184.

Krishna, B., K. Krishnappa, N. Reddy and M. Anjanappa. 1999. Effect of fertigation on growth and yield of carnation cultivars grown under poly house. Mysore J. Agric. Sci., 33(1): 33-38.

Madrid, R.A., A. Garcia and M. Boronat. 2001. Study of the Physico-Chemical parameters and nutrients of a soil cultivated under flood irrigation and fertigation, Agrochimica, 45(3): 99-107.

Mohammad, M. and S. Zuraiqi. 2003. Enhancement of yield and nitrogen use efficiencies by nitrogen drip-fertigation of garlic. J. Plant Nut., 26(9): 1749-1766.

Palai, S, M. Mishra and H. Mishra. 2002. Response of rose cv. Montezuma to different levels of N.P and K fertigation. Orissa J. Hort., 30(1): 51-53.

Raviv, M. and J. Blom. 2001. The effect of water availability and quality on photosynthesis and productivity of soillessgrown cut roses. Sci. Hort., 88: 257-276.

Synder, G. and E. Burt. 1976. Nitrogen fertilization of Bermuda grass turf through an irrigation system. J. Amer. Soc. Hort. Sci., 161(2): 145-148.

Umma, S. and J. Gowda. 1986. Effect of Nitrogen and pruning levels and their interaction on vase life of rose. Indian Rose Ann., 88-93.

Veerana, H., K. Abdul, G. Sujith and A. Khalak. 2001. Effect of fertigation and irrigation methods on yield, water and fertilizer use efficiency in Chilli (Capsicum annum L). South Indian Hort. 49: 101-104.

Young, P., W. Snyder, F. Martin and N. Hayslip. 1976. Rose response to NPK fertilization rates. Hort. Sci., 98(1): 109112.

Yusuf, N. and T. Dennis. 1999. Distribution of nutrient in cut flower roses and quantities of biomass and nutrients removed during harvest. Hort. Sci., 34(2): 251-253.

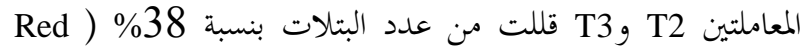

(Giant

وبنسبة 29\% (Sunflare)، علي التوالي. وهذه النتائج متماشية مع

ما وجده Ashok and Rengasamy (2000) حيث وجدوا إن أقصي عدد للبتلات في الوردة الواحدة كان في النباتات التي رويت بمياه ري محتوية علي 150 جزء في المليون من النتروجين. الخالاصة

يمكن ان نستخلص من هذا البحث أن كل صفات النمو والتطور

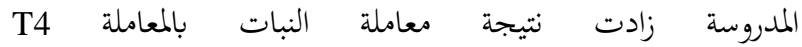
(175N+50P+190K ppm NPK fertigation)

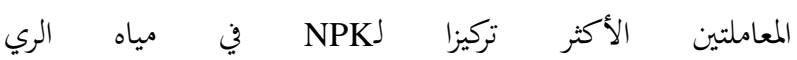
225N+70P+250K :T6 200N+60P+220K :T5) المليون) لها نفس التأثير ولكن التركيز الأمثل والاقتصادي بين كل المعاملات هي المعاملة 175N+50P+190K) T4 الذي يعطي إنتاج أكثر من المعاملات الاخري. تأثير المعامل (T3) قريب من تأثير المعاملة (T4) ومع ذلك يوجد فروق معنوية بين هاتين المعاملتين في كل الصفات التي درست وهذا هو السبب الذي ينصح باستخدام المعاملة T4 لنمو جيد وجودة جيدة للنبات لأصناف الورد تحت الدراسة.

\section{المراجع}

Armitage, A.M. and J.M. Laushman. 2003. Specialty Cut Flowers, 2nd Edition. Timber Press, Portland, OR. Pp. 636.

Armitage, A.M. and M. Jawjita. 1979. The effect of nitrogen concentration and supplement of light on the growth and quality of Caliente Roses. Hort. Sci., 14(5): 614-615.

Ashok, A., D. Arun and P. Rengasamy. 1999. Influence of graded levels and sources of $\mathrm{N}$ fertigation of cut rose cv. 'First Red' under protected conditions. South Indian Hort., 47(1-6): 115-118.

Ashok, A. and P. Rengasamy. 2000. Effect of $\mathrm{N}$ fertigation at different levels and sources on the growth of cut rose cv. 'First Red' under greenhouse conditions. South Indian Hort., 48(1-6): 139-141.

Bachmann, J. 2006. Specialty Cut Flower Production and Marketing. ATTRA Publication. P.O. Box. 3838. Butte, MT. 59702.

Bylov, V., Voronchikhina and G. Mikhallov. 1977. Application of complete soluble fertilizer in protected floriculture area. Bulleten Gloovnogo Botanicheskoga Soda; (105): 90-94. (Hort. Absts., 48(7): 6648, 1978). 


\title{
ABSTRACT \\ Effects of N.P.K. Fertigation on Growth and Development of Rose Cultivars under Glasshouse Condition
}

\author{
Mohammed Refdan Alhajhoj Al-Qahtani
}

The effects of six combinations of NPK fertigation $(100 \mathrm{~N}+20 \mathrm{P}+100 \mathrm{~K}(\mathrm{~T} 1), 125 \mathrm{~N}+30 \mathrm{P}+130 \mathrm{~K}(\mathrm{~T} 2), 150 \mathrm{~N}+40$ $\mathrm{P}+160 \mathrm{~K}(\mathrm{~T} 3), 175 \mathrm{~N}+50 \mathrm{P}+190 \mathrm{~K}(\mathrm{~T} 4), 200 \mathrm{~N}+60 \mathrm{P}+220 \mathrm{~K}$ (T5) and $\left.225 \mathrm{~N}+70 \mathrm{P}+250 \mathrm{~K}\left(\mathrm{~T}^{\wedge}\right) \mathrm{ppm}\right)$ at 3 days interval on plant growth and flowering in two rose cultivars: Red Giant and Sunflare were investigated. Plant height, number of branches per plant, number of leaves per plant, flowering time, number of flowers per plant and number of petals per flower were maximum with fertigation at $175 \mathrm{~N}+50 \mathrm{P}+190 \mathrm{~K} \mathrm{ppm}$.
All other combinations above this level of NPK produced more or less statistically similar results hence are not recommended as economical one. NPK fertigation combinations below $175 \mathrm{~N}+50 \mathrm{P}+190 \mathrm{~K}$ ppm level were statistically produced inferior plant growth characteristics hence not recommended as ideal ones.

Key Words: Rose, Rosa hybrida, N.P.K., Fertigation, Rose cultivars, Red Giant, sunflower 